\title{
THE MAXIMUM OF THE PERIODOGRAM FOR A HEAVY-TAILED SEQUENCE ${ }^{1}$
}

\section{By Thomas Mikosch, Sidney Resnick and Gennady Samorodnitsky \\ University of Groningen, Cornell University and Cornell University}

We consider the maximum of the periodogram based on an infinite variance heavy-tailed sequence. For $\alpha<1$ we show that the maxima constitute a weakly convergent sequence and find its limiting distribution. For $1 \leq \alpha<2$ we show that the sequence of the maxima is not tight and find a normalization that makes it tight.

1. Introduction. For a sequence $X=\left(X_{t}, t \geq 1\right)$ of random variables we consider its periodogram defined by

$$
\begin{aligned}
I_{n, X}(x) & =\left|J_{n, X}(x)\right|^{2}=\left|b_{n}^{-1} \sum_{t=1}^{n} X_{t} \exp (-i 2 \pi x t)\right|^{2} \\
& =\left(b_{n}^{-1} \sum_{t=1}^{n} X_{t} \cos (2 \pi x t)\right)^{2}+\left(b_{n}^{-1} \sum_{t=1}^{n} X_{t} \sin (2 \pi x t)\right)^{2}, \\
x & \quad x \in[0,0.5],
\end{aligned}
$$

where $\left(b_{n}\right)$ is an appropriate norming sequence to be specified later. We are interested in the limit behavior of the sequences

$$
M_{n, X}=\max _{x \in[0,0.5]} I_{n, X}(x) \text { and } \widetilde{M}_{n, X}=\max _{j=1, \ldots, q} I_{n, X}\left(\omega_{j}\right),
$$

for a stationary infinite variance sequence $X$. Here

$$
2 \pi \omega_{j}=2 \pi j / n, \quad j=1, \ldots, q, \quad q=q_{n}=\max \{j: 1 \leq j<n / 2\}
$$

denote the Fourier frequencies in $(0, \pi)$.

First we recall some results for finite and infinite variance sequences on the limit behavior of $I_{n, X}, M_{n, X}$ and $\widetilde{M}_{n, X}$. We commence with an iid sequence which we always denote by $Z=\left(Z_{t}\right)$. Assume for the moment that $E Z_{1}=0$ and $E Z_{1}^{2}=1$, and choose $b_{n}=n^{1 / 2}$. If the $Z_{t}$ 's are iid $N(0,1)$, then the vector of the periodogram ordinates

$$
I_{n, Z}\left(\omega_{j}\right), \quad j=1, \ldots, q,
$$

constitutes an iid standard exponential sequence. Hence the extreme value theory for these vectors is the well-known theory for the extremes of an iid

Received October 1997; revised August 1998.

${ }^{1}$ Supported in part by NSF Grant DMS-97-04982.

AMS 1991 subject classifications. Primary 62M15; secondary 60F05, 60G10, 60G55.

Key words and phrases. Periodogram, discrete Fourier transform, stable random variable, stable process, stochastic integral, infinite variance, linear process, point process convergence. 
exponential sequence. In particular,

$$
\widetilde{M}_{n, Z}-\ln q \Rightarrow Y,
$$

where $\Rightarrow$ denotes weak convergence and $Y$ has the standard Gumbel distribution $P[Y \leq x]=\exp \{-\exp \{-x\}\}, x \in \mathbb{R}$. For iid $N(0,1)$ random variables $Z_{t}$, Turkman and Walker (1984) showed that an analogue of (1.4) holds for $M_{n, Z}$ but the centering constants have to be slightly modified.

The quantity $\widetilde{M}_{n, Z}$ has been used in time series analysis for a long time; see Section 6.1.4 in Priestley (1981), who gives a historical account. The normalized statistic $\widetilde{M}_{n, Z}$, or its studentized version

$$
g=\widetilde{M}_{n, Z} / \sum_{j=1}^{q} I_{n, Z}\left(\omega_{j}\right),
$$

was used to construct asymptotic or exact tests based on the maximum of the periodogram for the null hypothesis that the $Z_{t}$ 's are iid $N(0,1)$. The perhaps best known result in this context was proved by Fisher (1929). He calculated the exact distribution of $g$ for iid $N(0,1) Z_{t}$ 's. Therefore, the expression $g$ is known as Fisher's g-statistic. If the $Z_{t}$ 's are not Gaussian, an exact result for the distribution of $g$ is difficult to obtain and therefore an asymptotic result such as (1.4) seems more appropriate. Davis and Mikosch (1999) showed that relation (1.4) remains valid if the $Z_{t}$ 's are iid non-Gaussian with $E Z_{1}=0$, $E Z_{1}^{2}=1$ and $E\left|Z_{1}\right|^{2+\delta}<\infty$ for some $\delta>0$. Thus the asymptotic relation (1.4) may serve as the theoretical basis for a large sample test based on the maximum of the periodogram for the null hypothesis that the $Z_{t}$ 's are iid with finite variance.

We note that the periodogram ordinates (1.3) have many properties in common with an iid standard exponential sequence, provided the second moment of the $Z_{t}$ 's is finite. This claim is supported by relation (1.4), but also by various other results. For example, Freedman and Lane (1980) proved that the empirical distribution function, constructed from (1.3), converges uniformly in probability to the exponential distribution function. Chen and Hannan (1980) extended this result from convergence in probability to convergence a.s. See Davis and Mikosch (1999) for more references to this phenomenon.

It is the aim of this paper to study the maximum of the periodogram for sequences of heavy-tailed random variables. In Section 2 we assume that $\left(Z_{t}, t \in \mathbb{Z}\right)$ is a sequence of iid random variables whose common distribution $F$ is in the domain of attraction of an $\alpha$-stable random variable where $0<\alpha<1$. (The definition of an $\alpha$-stable random variable is given below.) This means there exist $p, q \geq 0$ with $p+q=1$ and a slowly varying function $L(x)$, such that

$$
\lim _{x \rightarrow \infty} \frac{P\left[Z_{1}>x\right]}{P\left[\left|Z_{1}\right|>x\right]}=p, \quad \lim _{x \rightarrow \infty} \frac{P\left[Z_{1} \leq-x\right]}{P\left[\left|Z_{1}\right|>x\right]}=q
$$

and

$$
P\left[\left|Z_{1}\right|>x\right] \sim x^{-\alpha} L(x), \quad x \rightarrow \infty
$$


The normalizing sequence $\left(b_{n}\right)$ is defined via the quantile function of the distribution of $\left|Z_{1}\right|$, namely,

$$
b_{n}=\left(\frac{1}{P\left[\left|Z_{1}\right|>\cdot\right]}\right)^{\leftarrow}(n)
$$

In Section 3 we will assume $\left(Z_{t}\right)$ is an iid sequence of infinite variance symmetric $\alpha$-stable $(S \alpha S)$ random variables, $1 \leq \alpha<2$. Recall that a random variable $Y_{\alpha}$ is said to have a stable distribution $\left(Y_{\alpha} \sim S_{\alpha}(\sigma, \beta, \mu)\right)$ if there are parameters $0<\alpha \leq 2, \sigma \geq 0,-1 \leq \beta \leq 1$ and $\mu$ real such that its characteristic function has the form

$$
E \exp \left(i t Y_{\alpha}\right)= \begin{cases}\exp \left\{i \mu t-\sigma^{\alpha}|t|^{\alpha}(1-i \beta \operatorname{sign}(t) \tan (\pi \alpha / 2))\right\}, & \text { if } \alpha \neq 1 \\ \exp \{i \mu t-\sigma|t|(1+(2 i \beta / \pi) \operatorname{sign}(t) \ln |t|)\}, & \text { if } \alpha=1\end{cases}
$$

If $\beta=\mu=0$, then $Y_{\alpha}$ is $S \alpha S$. In this case, we set $b_{n}=n^{1 / \alpha}$ and for convenience we also assume in Section 3 that $Z_{1} \sim S_{\alpha}(1,0,0)$.

Recall that the heavy-tailed random variables considered in Sections 2 and 3 have infinite variance and, therefore, the limit theory for $I_{n, Z}$ and $M_{n, Z}$ is totally different from the finite variance case. In particular, there is no asymptotic independence of the periodogram ordinates. An indication of this fact is provided by a result of Freedman and Lane (1981) who showed that the empirical distribution function of the sequence (1.3) converges weakly to a random nondegenerate limit. This is in contrast to the aforementioned result in the finite variance case.

We remark that the random variables in the domain of attraction of an $\alpha$ stable law do not exhaust what can be termed "heavy-tailed" random variables. For example, one can have a heavy-tailed distribution whose tails are not balanced or whose tails are only bounded by Pareto tails. It is very likely that the behavior of $I_{n, Z}$ and $M_{n, Z}$ in such cases is very different from the finite variance case as well. However, it appears that precise limit theory can only be developed in the domain of attraction of a stable law and certainly the case under consideration is the only case likely to be relevent to statistical inference.

In the $S \alpha S$ case, Klüppelberg and Mikosch (1993) studied convergence of the finite-dimensional distributions of the process $\left(I_{n, Z}(x)\right)_{x \in[0,0.5]}$. In particular, they showed that

$$
\left(I_{n, Z}\left(x_{j}\right)\right)_{j=1, \ldots, m} \Rightarrow\left(\alpha^{2}\left(x_{j}\right)+\beta^{2}\left(x_{j}\right)\right)_{j=1, \ldots, m},
$$

where the $x_{j}$ 's are distinct frequencies in $(0,0.5)$ such that $1, x_{1}, \ldots, x_{m}$ are rationally independent. This means that $r_{0}+r_{1} x_{1}+\cdots+r_{m} x_{m}=0$ for rational $r_{i}$ 's implies that the $r_{i}$ 's must be zero. Clearly, the $x_{i}$ 's are then all irrational numbers. The limit vector

$$
\mathbf{A}_{m}=\left(\alpha\left(x_{1}\right), \beta\left(x_{1}\right), \ldots, \alpha\left(x_{m}\right), \beta\left(x_{m}\right)\right)
$$


has characteristic function

$$
\begin{aligned}
& E \exp \left[i\left(\mathbf{t}, \mathbf{A}_{m}\right)\right] \\
& =\exp \left\{-\int_{(0,1)^{m}}\left|\sum_{j=1}^{m}\left(t_{2 j-1} \cos \left(2 \pi y_{j}\right)+t_{2 j} \sin \left(2 \pi y_{j}\right)\right)\right|^{\alpha} d y\right\}, \\
& \mathbf{t} \in \mathbb{R}^{2 m} .
\end{aligned}
$$

Hence $\mathbf{A}_{m}$ is an $S \alpha S$ random vector in $\mathbb{R}^{2 m}$; see Samorodnitsky and Taqqu (1994), Section 2.1.

The limit result (1.8) turns out to be helpful for understanding the asymptotic behavior of $M_{n, Z}$ and $\widetilde{M}_{n, Z}$. When $\alpha \in(0,1)$ and the $Z_{t}$ 's are $S \alpha S$, it will be shown that both $\left(M_{n, Z}\right)$ and $\left(\widetilde{M}_{n, Z}\right)$ have the distributional limit $\max _{r \in I_{0}}\left(\alpha^{2}(x)+\beta^{2}(x)\right)$, where $I_{0} \subset(0,0.5)$ is a countable set of rationally independent numbers which is dense in $[0,0.5]$. This limit will also be identified as the weak limit of the sequence $\left(\left(b_{n}^{-1} \sum_{t=1}^{n}\left|Z_{t}\right|\right)^{2}\right)$, that is, the square of a positive $\alpha$-stable random variable. The case $\alpha \in[1,2)$ is more complicated. The sequence $\left(M_{n, Z}\right)$ is not tight, and so additional normalization is required. This is not surprising since the case $\alpha \in[1,2)$ may be considered as intermediate between the finite variance case [see (1.4)] and the infinite first moment case.

The asymptotic results for an iid heavy-tailed sequence $\left(Z_{t}, t \in \mathbb{Z}\right)$ are the theoretical basis for results on the weak limit behavior of $\left(M_{n, X}\right)$, where $X$ is a linear process given by

$$
X_{t}=\sum_{j=-\infty}^{\infty} \psi_{j} Z_{t-j}, \quad t \in \mathbb{Z} .
$$

One obviously needs certain assumptions on the coefficients $\psi_{j}$ for this series to converge. We will assume that for some $0<\epsilon<\alpha$,

$$
\sum_{j=-\infty}^{\infty}\left|\psi_{j}\right|^{\alpha-\epsilon}<\infty .
$$

This assumption is more than what is needed for the series in (1.10) to converge a.s. If $Z_{1}$ is in the domain of normal attraction of an $\alpha$-stable law, then we will only assume that

$$
\sum_{j=-\infty}^{\infty}\left|\psi_{j}\right|^{\alpha}<\infty .
$$

We use assumption (1.11) in Lemma 2.6 below. The situation here is similar to that when one wants to compare the tail distribution of an infinite linear combination of iid random variables with regularly varying tails with the tail distribution of one of these random variables. Unless the slowly varying function involved satisfies certain conditions, one has to assume a little more on the coefficients in the linear combination than necessary for convergence of the series. Also in the present case, one can use assumption (1.12) instead 
of (1.11) in more cases than just in the domain of normal attraction: one only has to impose certain technical assumptions on the slowly varying function. We feel, however, that the resulting gain in generality does not justify the resulting loss in clarity of presentation.

In what follows, we present the result on the asymptotic behavior of the maximum of the periodogram both for an iid heavy-tailed sequence $Z$ and the corresponding linear process $X$ defined by (1.10). In Section 2 we give the complete solution to the limit problem in the case $\alpha \in(0,1)$. In Section 3 we deal with the tightness of the sequence of maxima of the periodogram in the case $\alpha \in[1,2)$ and the $Z_{t}$ 's are $S \alpha S$.

2. The case $\alpha \in(\mathbf{0}, \mathbf{1})$. In this section, $Z=\left(Z_{t}\right)$ is a sequence of iid heavytailed random variables satisfying (1.6) and (1.7) for some $\alpha \in(0,1)$. In what follows, we consider three independent sequence $\left(\Gamma_{j}\right),\left(U_{j}\right)$ and $\left(B_{j}\right)$, defined on the same probability space. The first one, $\left(\Gamma_{j}\right)$, is the arrival sequence of a unit rate Poisson process on $\mathbb{R}_{+}$. The random variables $U_{j}$ are iid $U(0,1)$, and the $B_{j}$ 's are iid satisfying

$$
P\left[B_{1}=1\right]=p \quad \text { and } \quad P\left[B_{1}=-1\right]=q,
$$

where $p$ and $q$ are defined in (1.6).

Recall the definition of $M_{n, Z}$ and $\widetilde{M}_{n, Z}$ from (1.2). The following result completely characterizes the distributional limit of the maximum of the periodogram $I_{n, Z}$.

THEOREM 2.1. For $\alpha \in(0,1)$, the limit relations

$$
M_{n, Z} \Rightarrow Y_{\alpha}^{2} \quad \text { and } \quad \widetilde{M}_{n, Z}=\max _{j=1, \ldots, q} I_{n, Z}\left(\omega_{j}\right) \Rightarrow Y_{\alpha}^{2}
$$

hold, where $Y_{\alpha}=\sum_{j=1}^{\infty} \Gamma_{j}^{-1 / \alpha} \sim S_{\alpha}\left(C_{\alpha}^{-1 / \alpha}, 1,0\right)$ with

$$
C_{\alpha}=\left(\int_{0}^{\infty} x^{-\alpha} \sin x d x\right)^{-1}
$$

The proof rests on the following proposition. First, recall the definition of $J_{n, Z}$ from (1.1).

Proposition 2.2. For $0<\alpha<1$, the limit relation

$$
J_{n, Z}(x / n) \Rightarrow J_{\infty}(x):=\sum_{j=1}^{\infty} B_{j} \Gamma_{j}^{-1 / \alpha} \exp \left(-2 \pi i x U_{j}\right)
$$

as $n \rightarrow \infty$, holds in $C[0, \infty)$.

Proof. Let $M_{p}(\mathbb{E})$ be the set of point measures on

$$
\mathbb{E}:=[0,1] \times([-\infty, \infty] \backslash\{0\}),
$$


topologized by vague convergence: compare Resnick (1987). A typical element of $M_{p}(\mathbb{E})$ is represented by

$$
\sum_{k} \epsilon_{\mathbf{x}_{k}}(\cdot),
$$

where $\mathbf{x}_{k} \in \mathbb{E}$. The basic result we need is from Proposition 3.21 of Resnick (1987):

$$
N_{n}:=\sum_{k=1}^{n} \epsilon_{\left(k / n, Z_{k} / b_{n}\right)} \Rightarrow N:=\sum_{j=1}^{\infty} \epsilon_{\left(U_{j}, B_{j} \Gamma_{j}^{-1 / \alpha}\right)}
$$

in $M_{p}(\mathbb{E})$.

Now pick $\eta>0$ and define

$$
T_{\eta}: M_{p}(\mathbb{E}) \mapsto C[0, \infty)
$$

in the following way. If

$$
m=\sum_{j} \epsilon_{\left(t_{j}, v_{j}\right)} \in M_{p}(\mathbb{E})
$$

and all $v_{j}$ 's are finite, then

$$
\left(T_{\eta} m\right)(x)=\sum_{k} v_{j} 1_{\left[\left|v_{j}\right|>\eta\right]} \exp \left(-2 \pi i x t_{j}\right) .
$$

Otherwise, set $\left(T_{\eta} m\right)(x) \equiv 0$.

LEMMA 2.3. The map

$$
T_{\eta}: M_{p}(\mathbb{E}) \mapsto C[0, \infty)
$$

is continuous a.s. with respect to the distribution of $N$.

PROOF. It suffices to show that $x_{n} \rightarrow x \geq 0$ and $m_{n} \rightarrow^{v} m$ in $M_{p}(\mathbb{E})$, where

$$
m\{\partial([0,1] \times\{|v| \geq \eta\}) \cap[0,1] \times\{-\infty, \infty\}\}=0,
$$

$[\partial(A)$ is the boundary of any set $A]$ implies

$$
\left(T_{\eta} m_{n}\right)\left(x_{n}\right) \rightarrow\left(T_{\eta} m\right)(x) .
$$

To do this denote

$$
m_{n}=\sum_{j} \epsilon_{\left(t_{j}^{(n)}, v_{j}^{(n)}\right)} \quad \text { and } \quad m=\sum_{j} \epsilon_{\left[t_{j}, v_{j}\right)} .
$$

The set

$$
K_{\eta}:=[0,1] \times\{v:|v| \geq \eta\}
$$

is compact in $\mathbb{E}$ with $m\left(\partial K_{\eta}\right)=0$. For $n \geq n_{0}$,

$$
m_{n}\left(K_{\eta}\right)=m\left(K_{\eta}\right)=: l \text {, }
$$


say, and there is an enumeration of the points in $K_{\eta}$ such that

$$
\left(\left(t_{k}^{(n)}, v_{k}^{(n)}\right), 1 \leq k \leq l\right) \rightarrow\left(\left(t_{k}, v_{k}\right), 1 \leq k \leq l\right)
$$

and in fact, without loss of generality we may assume for given $\xi>0$ that

$$
\sup _{n \geq n_{0}}\left|x_{n}\right| \vee \sup _{k=1, \ldots, l}\left|v_{k}^{(n)}\right| \leq \xi
$$

Therefore,

$$
\begin{aligned}
& \left|\left(T_{\eta} m_{n}\right)\left(x_{n}\right)\right|-\left|\left(T_{\eta} m\right)(x)\right| \\
& \quad=\left|\sum_{k=1}^{l} v_{l}^{(n)} \exp \left(-2 \pi i x_{n} t_{k}^{(n)}\right)-\sum_{k=1}^{l} v_{k} \exp \left(-2 \pi i x t_{k}\right)\right| \\
& \quad \leq \sum_{k=1}^{l}\left|v_{k}^{(n)} \exp \left(-2 \pi i x_{n} t_{k}^{(n)}\right)-v_{k} \exp \left(-2 \pi i x t_{k}\right)\right| .
\end{aligned}
$$

Now

$$
\begin{aligned}
\left|v_{k}^{(n)} \exp \left(-2 \pi i x_{n} t_{k}^{(n)}\right)-v_{k} \exp \left(-2 \pi i x t_{k}\right)\right| \\
=\mid v_{k}^{(n)} \exp \left(-2 \pi i x_{n} t_{k}^{(n)}\right)-v_{k} \exp \left(-2 \pi i x_{n} t_{k}^{(n)}\right) \\
\quad+v_{k} \exp \left(-2 \pi i x_{n} t_{k}^{(n)}\right)-v_{k} \exp \left(-2 \pi i x t_{k}\right) \mid \\
\leq\left|v_{k}^{(n)}-v_{k}\right|+\left|v_{k}\right|\left|\exp \left(-2 \pi i x_{n} t_{k}^{(n)}\right)-\exp \left(-2 \pi i x t_{k}\right)\right| .
\end{aligned}
$$

Thus

$$
\begin{aligned}
& \left|\left(T_{\eta} m\right)_{n}\left(x_{n}\right)-\left(T_{\eta} m\right)(x)\right| \\
& \quad \leq \sum_{k=1}^{l}\left|v_{k}^{(n)}-v_{k}\right|+\sum_{k=1}^{l}\left|v_{k}\right|\left|\exp \left(-2 \pi i x_{n} t_{k}^{(n)}\right)-\exp \left(-2 \pi i x t_{k}\right)\right|,
\end{aligned}
$$

so

$$
\lim _{n \rightarrow \infty}\left|\left(T_{\eta} m_{n}\right)\left(x_{n}\right)-\left(T_{\eta} m\right)(x)\right|=0 .
$$

This completes the proof of Lemma 2.3.

We now continue with the proof of Proposition 2.2. We apply the functional $T_{\eta}$ to both sides in (2.4) to obtain

$$
\begin{aligned}
& J_{n}^{(\eta)}(x / n):=\sum_{j=1}^{n} \frac{Z_{j}}{b_{n}} \exp (-2 \pi i x j / n) 1_{\left[\left|Z_{j}\right|>\eta b_{n}\right]} \\
& \Rightarrow \sum_{j=1}^{\infty} B_{j} \Gamma_{j}^{-1 / \alpha} \exp \left(-2 \pi i x U_{j}\right) 1_{\left[\Gamma_{j}^{-1 / \alpha}>\eta\right]}:=J_{\infty}^{(\eta)}(x)
\end{aligned}
$$


in $C[0, \infty)$. Also, as $\eta \rightarrow 0$ we have

$$
J_{\infty}^{(\eta)}(x) \Rightarrow J_{\infty}(x):=\sum_{j=1}^{\infty} B_{j} \Gamma_{j}^{-1 / \alpha} \exp \left(-2 \pi i x U_{j}\right)
$$

and so from Theorem 4.2 of Billingsley (1968), page 25, it remains to prove for any $\theta>0$,

$$
\lim _{\eta \rightarrow 0} \limsup _{n \rightarrow \infty} P\left[\left\|J_{n, Z}^{(\eta)}-J_{n, Z}\right\|>\theta\right]=0,
$$

where $\|x(\cdot)-y(\cdot)\|$ is the $C[0, \infty)$ metric distance between $x, y \in C[0, \infty)$. The method of proof of (2.6) will be amply demonstrated if we show for any $\theta>0$,

(2.7) $\lim _{\eta \rightarrow 0} \limsup _{n \rightarrow \infty} P\left[\sup _{0 \leq x \leq 1}\left|\sum_{j=1}^{n} \frac{Z_{j}}{b_{n}} \exp (-2 \pi i x j / n) 1_{\left[\left|Z_{j}\right| \leq \eta b_{n}\right]}\right|>\theta\right]=0$.

The expression in (2.7) has a bound,

$\lim _{\eta \rightarrow 0} \limsup _{n \rightarrow \infty} P\left[\sum_{j=1}^{n}\left|\frac{Z_{j}}{b_{n}}\right| 1_{\left[\left|Z_{j}\right| \leq \eta b_{n}\right]}>\theta\right] \leq \lim _{\eta \rightarrow 0} \limsup _{n \rightarrow \infty} n E\left(\left|\frac{Z_{1}}{b_{n}}\right| 1_{\left[\left|Z_{1}\right| \leq b_{n} \eta\right]}\right) / \theta$,

and applying Karamata's theorem in the form given, for example, on page 579 of Feller (1971), we get the bound

$$
\lim _{\eta \rightarrow 0} \int_{\{x:|x| \leq \eta\}}|x| \nu(d x) / \theta=0
$$

where

$$
\nu(d x)=p \alpha x^{-\alpha-1} d x 1_{[x>0]}+q \alpha|x|^{-\alpha-1} d x 1_{[x<0]} .
$$

This completes the proof of Proposition 2.2.

We now continue with the proof of Theorem 2.1. We start with the proof of the first part of (2.1). It is immediate that

$$
M_{n, Z} \leq\left(b_{n}^{-1} \sum_{t=1}^{n}\left|Z_{t}\right|\right)^{2}
$$

It is well known [cf. Feller (1971)] that

$$
b_{n}^{-1} \sum_{t=1}^{n}\left|Z_{t}\right| \Rightarrow Y_{\alpha}=\sum_{j=1}^{\infty} \Gamma_{j}^{-1 / \alpha} \sim S_{\alpha}\left(C_{\alpha}^{-1 / \alpha}, 1,0\right)
$$

Hence the sequence $\left(M_{n, Z}\right)$ is stochastically bounded and it remains to show the lower bound in the limit for the maximum of the periodogram.

It suffices to show

$$
\liminf _{n \rightarrow \infty} P\left[M_{n, Z}>\gamma\right] \geq P\left[Y_{\alpha}^{2}>\gamma\right]
$$


since then we would have

$$
\begin{aligned}
P\left[Y_{\alpha}^{2}>\gamma\right] & \leq \liminf _{n \rightarrow \infty} P\left[M_{n, Z}>\gamma\right] \leq \limsup _{n \rightarrow \infty} P\left[M_{n, Z}>\gamma\right] \\
& \leq \limsup _{n \rightarrow \infty} P\left[\left(\sum_{j=1}^{n}\left|\frac{Z_{j}}{b_{n}}\right|\right)^{2}>\gamma\right]=P\left[Y_{\alpha}^{2}>\gamma\right] .
\end{aligned}
$$

But for any $T>0$ and $n \geq 2 T$,

$$
\begin{aligned}
M_{n, Z} & =\sup _{x \in[0,1 / 2]}\left|J_{n, Z}(x)\right|^{2}=\sup _{x \in[0, n / 2]}\left|J_{n, Z}(x / n)\right|^{2} \\
& \geq \sup _{x \in[0, T]}\left|J_{n, Z}(x / n)\right|^{2} \Rightarrow \sup _{x \in[0, T]}\left|J_{\infty}(x)\right|^{2},
\end{aligned}
$$

and therefore,

$$
\begin{aligned}
\liminf _{n \rightarrow \infty} P\left[M_{n, Z}>\gamma\right] & \geq \liminf _{n \rightarrow \infty} P\left[\sup _{x \in[0, T]}\left|J_{n, Z}(x / n)\right|^{2}>\gamma\right] \\
& =P\left[\sup _{x \in[0, T]}\left|J_{\infty}(x)\right|^{2}>\gamma\right] .
\end{aligned}
$$

This is true for any $T$, so we obtain

$$
\liminf _{n \rightarrow \infty} P\left[M_{n, Z}>\gamma\right] \geq P\left[\sup _{x \in[0, \infty)}\left|J_{\infty}(x)\right|^{2}>\gamma\right] .
$$

The following lemma identifies the distribution of $\sup _{x \in[0, \infty)}\left|J_{\infty}(x)\right|^{2}$.

LEMMA 2.4. We have

$$
\begin{aligned}
\sup _{x \in[0, \infty)}\left|J_{\infty}(x)\right|^{2} & =\sup _{x \in[0, \infty)}\left|\sum_{j=1}^{\infty} B_{j} \Gamma_{j}^{-1 / \alpha} \exp \left(-2 \pi i x U_{j}\right)\right|^{2} \\
& =Y_{\alpha}^{2}=\left(\sum_{j=1}^{\infty} \Gamma_{j}^{-1 / \alpha}\right)^{2}
\end{aligned}
$$

PROOF. It is enough to prove that the right-hand side of (2.11) is a lower bound for the left-hand side, the other bound being trivial. Define

$$
\begin{array}{r}
\Omega_{+}=\left\{\omega: \sum_{j=1}^{\infty} \Gamma_{j}^{-1 / \alpha}(\omega)<\infty \text { and for every } m \geq 1\right. \text { the numbers } \\
\left.\left(U_{1}(\omega), \ldots, U_{m}(\omega)\right) \text { are rationally independent }\right\} .
\end{array}
$$

Then $P\left[\Omega_{+}\right]=1$ and for any $\omega \in \Omega_{+}$, by the result of Weyl (1916), the family of the $m$-tuples

$$
\left\{\left(\left\{x U_{1}(\omega)\right\}, \ldots,\left\{x U_{m}(\omega)\right\}\right), x \geq 0\right\}
$$

(where $\{z\}$ denotes the fractional part of $z$ ) is dense in $[0,1]^{m}$. 
Fix an element $\omega \in \Omega_{+}$and take any $\varepsilon>0$. Choose an $N \geq 1$ such that $\sum_{j=N+1}^{\infty} \Gamma_{j}^{-1 / \alpha}(\omega)<\varepsilon$. There is an $x_{0} \in[0, \infty)$ such that

$$
\Re\left(B_{j} \exp \left(-2 \pi i x_{0} U_{j}\right)\right) \geq 1-\frac{\varepsilon}{N \Gamma_{j}^{-1 / \alpha}}, \quad j=1, \ldots, N .
$$

We then have

$$
\begin{aligned}
& \sup _{x \in[0, \infty)}\left|\sum_{j=1}^{\infty} B_{j} \Gamma_{j}^{-1 / \alpha} \exp \left(-2 \pi i x U_{j}\right)\right| \\
& \geq \sup _{x \in[0, \infty)}\left|\sum_{j=1}^{N} B_{j} \Gamma_{j}^{-1 / \alpha} \exp \left(-2 \pi i x U_{j}\right)\right|-\sum_{j=N+1}^{\infty} \Gamma_{j}^{-1 / \alpha} \\
& \geq\left|\sum_{j=1}^{N} B_{j} \Gamma_{j}^{-1 / \alpha} \exp \left(-2 \pi i x_{0} U_{j}\right)\right|-\varepsilon \geq \Re\left(\sum_{j=1}^{N} B_{j} \Gamma_{j}^{-1 / \alpha} \exp \left(-2 \pi i x U_{j}\right)\right)-\varepsilon \\
& \geq \sum_{j=1}^{N}\left(1-\frac{\varepsilon}{N \Gamma_{j}^{-1 / \alpha}}\right) \Gamma_{j}^{-1 / \alpha}-\varepsilon=\sum_{j=1}^{N} \Gamma_{j}^{-1 / \alpha}-2 \varepsilon .
\end{aligned}
$$

Letting first $N \rightarrow \infty$ and then $\varepsilon \rightarrow 0$, we obtain (2.11). This proves the lemma and completes the derivation of the limit distribution for $M_{n, Z}$.

Thus it remains to prove $\widetilde{M}_{n, Z} \Rightarrow Y_{\alpha}^{2}$. In view of $M_{n, Z} \Rightarrow Y_{\alpha}^{2}$ it suffices to show that for all $\gamma>0$,

$$
\liminf _{n \rightarrow \infty} P\left[\widetilde{M}_{n, Z}>\gamma\right] \geq P\left[Y_{\alpha}^{2}>\gamma\right] .
$$

We observe that for any integer $K$ and sufficiently large $n$,

$$
P\left[\sup _{j=1, \ldots, q}\left|J_{n}(j / n)\right|^{2}>\gamma\right] \geq P\left[\sup _{j=1, \ldots, K}\left|J_{n}(j / n)\right|^{2}>\gamma\right] .
$$

Now from Proposition 2.2 we have

$$
\left(J_{n}(j / n), 1 \leq j \leq K\right) \Rightarrow\left(J_{\infty}(j), 1 \leq j \leq K\right)
$$

in $\mathbb{R}^{K}$, hence

$$
\sup _{j=1, \ldots, K}\left|J_{n}(j / n)\right|^{2} \Rightarrow \sup _{j=1, \ldots, K}\left|J_{\infty}(j)\right|^{2}
$$

and so

$$
\liminf _{n \rightarrow \infty} P\left[\sup _{j=1, \ldots, q}\left|J_{n}(j / n)\right|^{2}>\gamma\right] \geq P\left[\sup _{j=1, \ldots, \infty}\left|J_{\infty}(j)\right|^{2}>\gamma\right] .
$$

Finish the proof by adapting Lemma 2.4 to this case [in particular, it follows from Weyl (1916) that the family of the $m$-tuples

$$
\left\{\left(\left\{j U_{1}(\omega)\right\}, \ldots,\left\{j U_{m}(\omega)\right\}\right), j=1,2, \ldots\right\}
$$

is dense in $[0,1]^{m}$ as well.] 
From the previous proof it is clear that the limit random variable $Y_{\alpha}^{2}$ appears as the weak limit of the sequence $\left(\left(b_{n}^{-1} \sum_{t=1}^{n}\left|Z_{t}\right|\right)^{2}\right)$. It also appears as $\sup _{x \geq 0}\left|J_{\infty}(x)\right|^{2}$. When $\left(Z_{t}\right)$ is a sequence of $S \alpha S$ variables, $Y_{\alpha}^{2}$ can also appear naturally in a third form which we describe in the following proposition.

Proposition 2.5. Let $\left(Z_{t}\right)$ be a sequence of iid $S \alpha S$ variables. Then the limit variable $Y_{\alpha}^{2}$ in (2.1) has representation

$$
Y_{\alpha}^{2} \stackrel{d}{=} \sup _{x \in I_{0}}\left(\alpha^{2}(x)+\beta^{2}(x)\right)
$$

where $I_{0} \subset(0,0.5)$ is a countable set such that, for an enumeration $\left(x_{n}\right)$ of $I_{0}$, for every $m \geq 1$ and $x_{1}, \ldots, x_{m} \in I_{0}$, the numbers $1, x_{1}, \ldots, x_{m}$ are rationally independent, $I_{0}$ is dense in $[0,0.5]$, and $\left((\alpha(x), \beta(x)), x \in I_{0}\right)$ is an exchangeable $S \alpha S$ process with finite-dimensional distributions determined by (1.9).

This represents $Y_{\alpha}^{2}$ as the result of two different limiting procedures. In Proposition 2.2, we normalize the argument by dividing by $n$ and consider $J_{n, Z}(x / n)$. It is also possible to proceed using a normalization as in (1.8) which does not divide the argument by $n$. This alternative representation of the limiting random variable tied to (1.8) and (1.9) will be used in Section 3.

PRoOF. Let $I_{0}$ be a subset of irrational numbers in $(0,0.5)$, having the properties given in the proposition. Let $\left(\Omega^{\prime}, \mathscr{F}^{\prime}, P^{\prime}\right)$ be a probability space rich enough to support a family of iid uniform $U(0,1)$ random variables $\left(U_{x}, x \in I_{0}\right)$. Further, suppose $M$ is an $S \alpha S$ random measure on $\left(\Omega^{\prime}, \mathscr{F}^{\prime}\right)$ with control measure $P^{\prime}$; compare Samorodnitsky and Taqqu (1994), Section 3.3.

Consider an $S \alpha S$ process $W$ defined by

$$
W(x)=\int_{\Omega^{\prime}} \exp \left(-2 \pi i U_{x}\left(\omega^{\prime}\right)\right) M\left(d \omega^{\prime}\right), \quad x \in I_{0} .
$$

It is immediate that for any distinct numbers $x_{1}, \ldots, x_{m} \in I_{0}$ and $m \geq 1$,

$$
\mathbf{B}_{m}=\left(\Re\left(W\left(x_{1}\right)\right), \mathfrak{s}\left(W\left(x_{1}\right)\right), \ldots, \mathfrak{R}\left(W\left(x_{m}\right)\right), \mathfrak{s}\left(W\left(x_{m}\right)\right)\right) \stackrel{d}{=} \mathbf{A}_{m},
$$

where $\mathbf{A}_{m}$ has characteristic function (1.9). Therefore, when $\left(Z_{t}\right)$ is a sequence of $S \alpha S$ variables, we have

$$
\liminf _{n \rightarrow \infty} P\left[M_{n, Z}>\gamma\right] \geq P\left[\sup _{x \in I_{0}}|W(x)|^{2}>\gamma\right]
$$

for all $\gamma \geq 0$.

The $S \alpha S$ process $W$ has a series representation [cf. Samorodnitsky and Taqqu (1994), Section 3.10]

$$
W(x)=C_{\alpha}^{1 / \alpha} \sum_{j=1}^{\infty} \varepsilon_{j} \Gamma_{j}^{-1 / \alpha} \exp (-2 \pi i U(x, j)), \quad x \in I_{0},
$$

where $\left(U(x, j) ; j \geq 1, x \in I_{0}\right)$ is an array of iid $U(0,1)$ random variables, $\left(\varepsilon_{j}\right)$ is a sequence of iid Rademacher random variables $\left(P\left[\varepsilon_{j}=1\right]=P\left[\varepsilon_{j}=-1\right]=\right.$ $0.5)$, and $\left(\Gamma_{j}\right)$ are the points of a unit rate Poisson process on $\mathbb{R}_{+}$. Moreover, 
these three sequences of random variables are independent. The representation (2.16) has to be interpreted in distribution, but, since we are interested only in distributional results, we may, as well, assume that it is an almost sure representation of $W$.

An argument very similar to that of Lemma 2.4 shows that

$$
\sup _{x \in I_{0}}|W(x)| \stackrel{d}{=} C_{\alpha}^{1 / \alpha} \sum_{j=1}^{\infty} \Gamma_{j}^{-1 / \alpha} \stackrel{d}{=} Y_{\alpha} .
$$

Therefore, it follows from (2.14) that

$$
\liminf _{n \rightarrow \infty} P\left[M_{n, Z}>\gamma\right] \geq P\left[Y_{\alpha}^{2}>\gamma\right]
$$

thus providing an alternative argument to Theorem 2.1 when $\left(Z_{t}\right)$ are $S \alpha S$.

Now we consider the periodogram of a general linear process $X=\left(X_{t}\right)$, given by (1.10), with the $Z_{t}$ 's satisfying (1.6) and (1.7) as before and whose coefficients $\psi_{j}$ satisfy $(1.11)$ or $(1.12)$, if the $Z_{t}$ 's are in the domain of the normal attraction of the stable. Write

$$
\psi(x)=\sum_{j=-\infty}^{\infty} \psi_{j} \exp (-i 2 \pi x j), x \in[0,0.5]
$$

for the transfer function of the linear filter $\left(\psi_{j}\right)$. Notice that the power transfer function

$$
f_{X}(x)=|\psi(x)|^{2}
$$

is the spectral density of a Gaussian linear process with representation (1.10), where the $Z_{t}$ 's have to be replaced by mean-zero iid Gaussian random variables.

LEMMA 2.6. Assume that $f_{X}$ is strictly positive on $[0,0.5]$. Then under the assumptions of Theorem 2.1,

$$
\max _{x \in[0,0.5]}\left|I_{n, X}(x) / f_{X}(x)-I_{n, Z}(x)\right| \stackrel{P}{\rightarrow} 0 .
$$

Proof. The following decomposition is standard [see, e.g., Brockwell and Davis (1991), page 346]:

$$
\begin{aligned}
I_{n, X}(x) & =\left|J_{n, X}(x)\right|^{2}=f_{X}(x)\left|J_{n, Z}(x)\right|^{2}+R_{n}(x) \\
& =f_{X}(x) I_{n, Z}(x)+R_{n}(x),
\end{aligned}
$$


where

$$
\begin{aligned}
R_{n}(x) & =\psi(x) J_{n, Z}(x) Y_{n}(-x)+\psi(-x) J_{n, Z}(-x) Y_{n}(x)+\left|Y_{n}(x)\right|^{2}, \\
J_{n, X}(x) & =b_{n}^{-1} \sum_{t=1}^{n} X_{t} \exp (-i 2 \pi x t) \\
& =b_{n}^{-1} \sum_{j=-\infty}^{\infty} \psi_{j} \exp (-i 2 \pi x j)\left(\sum_{t=1}^{n} Z_{t} \exp (-i 2 \pi x t)+V_{n, j}\right) \\
& =\psi(x) J_{n, Z}(x)+Y_{n}(x), \\
V_{n, j} & =\sum_{t=1-j}^{n-j} Z_{t} \exp (-i 2 \pi x t)-\sum_{t=1}^{n} Z_{t} \exp (-i 2 \pi x t) \\
Y_{n}(x) & =b_{n}^{-1} \sum_{j=-\infty}^{\infty} \psi_{j} \exp (-i 2 \pi x j) V_{n, j} .
\end{aligned}
$$

The sequence

$$
M_{n, Z}=\max _{x \in[0,0.5]} I_{n, Z}(x), \quad n \geq 1,
$$

is tight and $f_{X}$ is bounded away from 0 and $\infty$. In view of (2.20), it suffices to show that $\max _{x \in[0,0.5]}\left|Y_{n}(x)\right| \rightarrow^{P} 0$. In what follows, we indicate the main steps of the proof. For the sake of simplicity we also assume $\psi_{j}=0$ for $j<0$. Then

$$
\begin{aligned}
Y_{n}(x) & =b_{n}^{-1} \sum_{j=n+1}^{\infty} \psi_{j} \exp (-i 2 \pi x j) V_{n, j}+b_{n}^{-1} \sum_{j=1}^{n} \psi_{j} \exp (-i 2 \pi x j) V_{n, j} \\
& =S_{1}(x)+S_{2}(x) .
\end{aligned}
$$

Furthermore,

$$
\begin{aligned}
S_{1}(x)= & -J_{n, Z}(x) \sum_{j=n+1}^{\infty} \psi_{j} \exp (-i 2 \pi x j) \\
& +b_{n}^{-1} \sum_{j=n+1}^{\infty} \psi_{j} \exp (-i 2 \pi x j) \sum_{t=1-j}^{n-j} Z_{t} \exp (-i 2 \pi x t) \\
= & S_{11}(x)+S_{12}(x) .
\end{aligned}
$$

Now

$$
\max _{x \in[0,0.5]}\left|S_{11}(x)\right| \leq \max _{x \in[0,0.5]}\left|J_{n, Z}(x)\right| \sum_{j=n+1}^{\infty}\left|\psi_{j}\right| \stackrel{P}{\rightarrow} 0
$$

and for $S_{12}(x)$ we have

$$
\left|S_{12}(x)\right| \leq b_{n}^{-1} \sum_{t=-n}^{-1}\left|Z_{t}\right| \sum_{j=n+1}^{n-t}\left|\psi_{j}\right|+b_{n}^{-1} \sum_{t=-\infty}^{-n-1}\left|Z_{t}\right| \sum_{j=1-t}^{n-t}\left|\psi_{j}\right| .
$$


Notice that

$$
\sum_{t=-n}^{-1} \frac{\left|Z_{t}\right|}{b_{n}} \sum_{j=n+1}^{n-t}\left|\psi_{j}\right| \stackrel{d}{=} \sum_{l=1}^{n} \frac{\left|Z_{l}\right|}{b_{n}} \sum_{j=n+1}^{n+l}\left|\psi_{j}\right| \stackrel{P}{\rightarrow} 0
$$

since $b_{n}^{-1} \sum_{l=1}^{n}\left|Z_{l}\right| \Rightarrow Y_{\alpha}$ and, by (1.11),

$$
\sum_{j=n+1}^{\infty}\left|\psi_{j}\right| \rightarrow 0
$$

The second expression in (2.21) is handled as follows. Write

$$
\begin{aligned}
\phi_{n}(\lambda) & =E \exp \left\{-\lambda b_{n}^{-1} \sum_{t=-\infty}^{-n-1}\left|Z_{t}\right| \sum_{j=1-t}^{n-t}\left|\psi_{j}\right|\right\} \\
& =E \exp \left(T_{1}+\cdots+T_{n}\right),
\end{aligned}
$$

where $T_{j}=-\lambda b_{n}^{-1} \sum_{i=n+1}^{\infty}\left|Z_{i}\right|\left|\psi_{i+j}\right|, j=1, \ldots, n$. Observe that $T_{1}, \ldots, T_{n}$ are associated and hence

$$
E \exp \left(T_{1}+\cdots+T_{n}\right) \geq \prod_{j=1}^{n} E \exp \left(T_{j}\right) \geq\left(E \exp \left(T_{1}\right)\right)^{n}
$$

Let

$$
\phi(\lambda)=E \exp \left\{-\lambda\left|Z_{1}\right|\right\}
$$

so that by Karamata's Tauberian theorem [Feller (1971), page 471],

$$
-\log \phi(\lambda) \sim 1-\phi(\lambda) \sim \Gamma(1-\alpha) \lambda^{\alpha} L(1 / \lambda), \quad \lambda \downarrow 0 .
$$

We use now the Potter bounds [see, e.g. Proposition 0.8 in Resnick (1987)] to see that for large $n$ and some $c>0$,

$$
\begin{aligned}
E e^{T_{1}} & =\prod_{i=n+1}^{\infty} \phi\left(b_{n}^{-1} \lambda\left|\psi_{i}\right|\right)=\exp \left\{-\sum_{i=n+2}^{\infty}\left(-\log \phi\left(b_{n}^{-1} \lambda\left|\psi_{i}\right|\right)\right)\right\} \\
& \geq \exp \left\{-c \frac{1}{n} \sum_{i=n+2}^{\infty} \frac{-\log \phi\left(b_{n}^{-1} \lambda\left|\psi_{i}\right|\right)}{-\log \phi\left(b_{n}^{-1} \lambda\right)}\right\} \\
& \geq \exp \left\{-c \frac{1}{n} \sum_{i=n+2}^{\infty}\left|\psi_{i}\right|^{(\alpha-\varepsilon)}\right\},
\end{aligned}
$$

from (2.24), and so by (2.22) and (2.23) we conclude that $\phi_{n}(\lambda) \rightarrow 1$. 
Now we turn to $S_{2}$ which we decompose as follows:

$$
\begin{aligned}
\left|S_{2}(x)\right|=\mid b_{n}^{-1} \sum_{j=1}^{n} \psi_{j} \exp (-i 2 \pi x j) \sum_{t=1-j}^{0} Z_{t} \exp (-i 2 \pi x t) \\
\quad-b_{n}^{-1} \sum_{j=1}^{n} \psi_{j} \exp (-i 2 \pi x j) \sum_{t=n-j+1}^{n} Z_{t} \exp (-i 2 \pi x t) \mid \\
\leq b_{n}^{-1} \sum_{j=1}^{n}\left|\psi_{j}\right|\left(\sum_{t=1-j}^{0}\left|Z_{t}\right|+\sum_{t=n-j+1}^{n}\left|Z_{t}\right|\right)=S_{21}+S_{22} .
\end{aligned}
$$

For the first double sum we have

$$
\begin{aligned}
S_{21} & =\sum_{j=1}^{n}\left|\psi_{j}\right| \sum_{t=1-j}^{0} \frac{\left|Z_{t}\right|}{b_{n}} \stackrel{d}{=} \sum_{j=1}^{n}\left|\psi_{j}\right| \sum_{k=1}^{j} \frac{\left|Z_{k}\right|}{b_{n}} \\
& =\sum_{k=1}^{k_{0}}\left(\sum_{j=k}^{n}\left|\psi_{j}\right|\right) \frac{\left|Z_{k}\right|}{b_{n}}+\sum_{k=k_{0}+1}^{n}\left(\sum_{j=k}^{n}\left|\psi_{j}\right|\right) \frac{\left|Z_{k}\right|}{b_{n}} \\
& =S_{211}+S_{212} .
\end{aligned}
$$

For fixed $k_{0}$,

$$
S_{211} \leq\left(\sum_{k=1}^{k_{0}} \frac{\left|Z_{k}\right|}{b_{n}}\right) \sum_{j=1}^{\infty}\left|\psi_{j}\right| \stackrel{P}{\rightarrow} 0
$$

and if $k_{0}$ is chosen so that $\sum_{k=k_{0}}^{\infty}\left|\psi_{k}\right|<\varepsilon$, then

$$
S_{212} \leq \varepsilon \sum_{k=k_{0}+1}^{n} \frac{\left|Z_{k}\right|}{b_{n}} \leq \epsilon \sum_{k=1}^{n} \frac{\left|Z_{k}\right|}{b_{n}} \Rightarrow \varepsilon Y_{\alpha} .
$$

Since $\epsilon>0$ is arbitrary, $S_{212} \rightarrow^{P} 0$. This proves that $S_{21} \rightarrow^{P} 0$.

For the second sum we have

$$
S_{22}=\sum_{t=1}^{n}\left|Z_{t}\right| \sum_{j=n-t+1}^{n}\left|\psi_{j}\right| \stackrel{d}{=} \sum_{t=1}^{n}\left|Z_{t}\right| \sum_{j=t}^{n}\left|\psi_{j}\right| .
$$

The sum on the right-hand side can be treated in a way similar to $S_{21}$. This completes the proof.

The following is now immediate from Lemma 2.6 and Theorem 2.1.

COROLlary 2.7. Assume that the iid sequence $\left(Z_{t}\right)$ satisfies (1.6) and (1.7) and that the coefficients $\psi_{j}$ of the linear process $\left(X_{t}\right)$ satisfy the condition (1.11) or, in the case of the domain of the normal attraction, (1.12). If $f_{X}$ is strictly positive on [0,0.5], then

$$
\max _{x \in[0,0.5]} I_{n, X}(x) / f_{X}(x) \Rightarrow Y_{\alpha}^{2} .
$$


We can also formulate the following limit result for Fisher's generalized $g$-statistic; see (1.5).

COROLlary 2.8. Under the assumptions of Corollary 2.7,

$$
\frac{\max _{j=1, \ldots, q} I_{n, X}\left(\omega_{j}\right) / f_{X}\left(\omega_{j}\right)}{\sum_{j=1}^{q} I_{n, X}\left(\omega_{j}\right) / f_{X}\left(\omega_{j}\right)} \Rightarrow \frac{Y_{\alpha}^{2}}{Y_{\alpha / 2}}
$$

where the mixed stable random vector $\left(Y_{\alpha}, Y_{\alpha / 2}\right)$ has Laplace transform

(2.26) $\quad E \exp \left\{-\lambda_{1} Y_{\alpha}-\lambda_{2} Y_{\alpha / 2}\right\}=\exp \left\{-E\left|\lambda_{1} C+\lambda_{2}^{1 / 2} G\right|^{\alpha}\right\}, \quad \lambda_{1}, \lambda_{2} \geq 0$.

Here $C$ and $G$ are independent random variables, $C$ is standard Cauchy and $G$ is $N(0,2)$. Alternatively, one can use

$$
\left(Y_{\alpha}, Y_{\alpha / 2}\right) \stackrel{d}{=}\left(\sum_{j=1}^{\infty} \Gamma_{j}^{-1 / \alpha}, \sum_{j=1}^{\infty} \Gamma_{j}^{-2 / \alpha}\right)
$$

where $\left(\Gamma_{j}, j \geq 1\right)$ are the standard Poisson arrival times.

REMARKS.

1. For $X=Z, f_{X}$ degenerates to a constant. Hence the left-hand side expression of (2.25) coincides with Fisher's g-statistic (1.5).

2. From the proof below it can be seen that $\max _{j=1, \ldots, q} I_{n, X}\left(\omega_{j}\right) / f_{X}\left(\omega_{j}\right)$ in (2.25) can be replaced with $\max _{x \in[0,0.5]} I_{n, X}(x) / f_{X}(x)$.

3. A more precise relationship between (2.26) and (2.27) is

$$
\left(Y_{\alpha}, Y_{\alpha / 2}\right) \stackrel{d}{=}\left(b \sum_{j=1}^{\infty} \Gamma_{j}^{-1 / \alpha}, b^{2} \sum_{j=1}^{\infty} \Gamma_{j}^{-2 / \alpha}\right)
$$

for some $b>0$. However, $b$ is immaterial in (2.25).

Proof. By Lemma 2.6, we have

$$
\sum_{j=1}^{q} I_{n, X}\left(\omega_{j}\right) / f_{X}\left(\omega_{j}\right)=\sum_{j=1}^{q} I_{n, Z}\left(\omega_{j}\right)\left(1+o_{P}(1)\right)=b_{n}^{-2} \sum_{t=1}^{n} Z_{t}^{2}\left(1+o_{P}(1)\right)
$$

and

$$
\max _{j=1, \ldots, q} I_{n, X}\left(\omega_{j}\right) / f_{X}\left(\omega_{j}\right)=\max _{j=1, \ldots, q} I_{n, Z}\left(\omega_{j}\right)\left(1+o_{P}(1)\right) .
$$

Hence it suffices to determine the weak limit of

$$
I_{n}=\frac{\max _{j=1, \ldots, q} I_{n, Z}\left(\omega_{j}\right)}{b_{n}^{-2} \sum_{t=1}^{n} Z_{t}^{2}} .
$$

We will prove the existence of a weak limit in (2.28) and the representation (2.27). The latter, and some algebra, imply (2.26). It follows from Theorem 2.1 that

$$
M_{n, Z}-\widetilde{M}_{n, Z} \stackrel{P}{\rightarrow} 0
$$


as $n \rightarrow \infty$. Therefore, we need to prove that

$$
\frac{\max _{x \in[0,0.5]}\left|b_{n}^{-1} \sum_{t=1}^{n} Z_{t} \exp (-i 2 \pi x t)\right|^{2}}{b_{n}^{-2} \sum_{t=1}^{n} Z_{t}^{2}} \Rightarrow \frac{Y_{\alpha}^{2}}{Y_{\alpha / 2}},
$$

where $\left(Y_{\alpha}, Y_{\alpha / 2}\right)$ is given by (2.27).

We observe, first, that the left-hand side of (2.29) is bounded from above by

$$
\frac{\left(b_{n}^{-1} \sum_{t=1}^{n}\left|Z_{t}\right|\right)^{2}}{b_{n}^{-2} \sum_{t=1}^{n} Z_{t}^{2}}
$$

and this self-normalized sum converges as in Resnick (1986). Therefore,

$$
\frac{\left(b_{n}^{-1} \sum_{t=1}^{n}\left|Z_{t}\right|\right)^{2}}{b_{n}^{-2} \sum_{t=1}^{n} Z_{t}^{2}} \Rightarrow \frac{\left(\sum_{j=1}^{\infty} \Gamma_{j}^{-1 / \alpha}\right)^{2}}{\sum_{j=1}^{\infty} \Gamma_{j}^{-2 / \alpha}} .
$$

On the other hand, for every $T>0$ and $n \geq 2 T$, the left-hand side of (2.29) is bounded from below by

$$
\frac{\max _{x \in[0, T]}\left|\int_{\{0<y \leq 1\}} u \exp (-i 2 \pi x y) N_{n}(d y, d u)\right|^{2}}{\int_{\{0<y \leq 1\}} u^{2} N_{n}(d y, d u)} .
$$

We repeat, once again, the truncation argument we used in the argument of Theorem 2.1, and the appeal to Theorem 4.2 in Billingsley (1968), to establish that the above ratio converges weakly to the corresponding ratio with $N_{n}$ replaced by $N$. After letting $T \rightarrow \infty$ and using the result of Weyl (1916), we obtain a lower bound that matches (2.30). We have, therefore, proved the existence of a weak limit in (2.28) and the representation (2.27).

3. The case $\alpha \in[1,2)$. In this section we assume $\left(Z_{t}\right)$ is an iid sequence of $S \alpha S$ random variables. If $\alpha \in[1,2)$ then the sequence $\left(M_{n, Z}\right)$ is not stochastically bounded. Indeed, the $S \alpha S$ process in (2.13) is well defined for all $0<\alpha<2$, and the argument used to get (2.15) works for any $0<\alpha<2$, and shows that for every $\gamma>0$,

$$
\liminf _{n \rightarrow \infty} P\left[M_{n, Z}>\gamma\right] \geq P\left[\sup _{x \in I_{0}}|W(x)|^{2}>\gamma\right]
$$

no matter what $\alpha$ is, and so our claim reduces to showing that

$$
\sup _{x \in I_{0}}|W(x)|=\infty \quad \text { with probability } 1 .
$$

To this end observe that, by symmetry, for every $N \geq 1$ and $\gamma>0$,

$$
\begin{aligned}
P\left[\sup _{x \in I_{0}}|W(x)|>\gamma\right] & \geq \frac{1}{2} P\left[\sup _{x \in I_{0}}\left|\sum_{j=1}^{N} \varepsilon_{j} \Gamma_{j}^{-1 / \alpha} \exp (i 2 \pi U(x, j))\right|>\gamma\right] \\
& =\frac{1}{2} P\left[\sum_{j=1}^{N} \Gamma_{j}^{-1 / \alpha}>\gamma\right] \rightarrow \frac{1}{2},
\end{aligned}
$$


where now $\left(\varepsilon_{j}\right)$ are iid symmetric Rademacher random variables taking values \pm 1 . In the last step we used again the argument in the proof of Lemma 2.4. Letting $N \rightarrow \infty$ and recalling that, for $1 \leq \alpha<2, \sum_{j=1}^{N} \Gamma_{j}^{-1 / \alpha} \rightarrow \infty$ with probability 1 , we see that

$$
P\left[\sup _{x \in I_{0}}|W(x)|>\gamma\right] \geq \frac{1}{2}
$$

for all $\gamma>0$. Since $W$ is an $S \alpha S$ process, it is bounded with probability 0 or 1 [see, e.g., Samorodnitsky and Taqqu (1994), Chapter 10], and so (3.1) follows.

Since the sequence $\left(M_{n, Z}\right)$ is not stochastically bounded when $\alpha \in[1,2)$, a normalization for $M_{n, Z}$ is needed. This is the content of the following result.

Proposition 3.1. If $\alpha \in(1,2)$, the sequence $\left(M_{n, Z} /(\ln n)^{(2 / \alpha)(\alpha-1)}, n \geq 2\right)$ is tight. If $\alpha=1$, the sequence $\left(M_{n, Z} /(\ln \ln n)^{2}, n>e\right)$ is tight.

Proof. The sequence $\left(Z_{j}\right)$ has a representation $\left(A_{j}^{1 / 2} G_{j}\right)$, where $\left(G_{j}\right)$ are iid $N(0,2)$ random variables and $\left(A_{j}\right)$ are iid positive $(\alpha / 2)$-stable random variables with Laplace transform $E \exp \left\{-\theta A_{1}\right\}=\exp \left\{-\theta^{\alpha / 2}\right\}, \theta \geq 0$. The two sequences are independent. See Samorodnitsky and Taqqu (1994), Section 1.3. Fix a $\delta>0$, write $A_{j}(\delta)=A_{j} I_{\left\{A_{j} \leq \delta n^{2 / \alpha}\right\}}$ and define

$$
I_{n, Z}^{-}(\delta)=\max _{x \in[0,0.5]}\left|n^{-1 / \alpha} \sum_{j=1}^{n} G_{j}\left(A_{j}(\delta)\right)^{1 / 2} \exp (-i 2 \pi x j)\right|^{2} .
$$

Given $\left(A_{j}\right)$, we introduce a pseudometric functional on $[0,0.5]$ :

$$
\begin{aligned}
d(x, y) & =\left(\sum_{j=1}^{n}\left|\left(A_{j}(\delta)\right)^{1 / 2} \exp (-i 2 \pi x j)-\left(A_{j}(\delta)\right)^{1 / 2} \exp (-i 2 \pi y j)\right|^{2}\right)^{1 / 2} \\
& =2\left(\sum_{j=1}^{n} A_{j}(\delta) \sin ^{2}(\pi(x-y) j)\right)^{1 / 2} .
\end{aligned}
$$

Write

$$
M_{n}(A(\delta))=\max _{j=1, \ldots, n} A_{j}(\delta) \text { and } S_{n}(A(\delta))=\sum_{j=1}^{n} A_{j}(\delta) .
$$

Then the pseudometric space $([0,0.5], d)$ has diameter

$$
D=\max _{x, y \in[0,0.5]} d(x, y) \leq 2\left(S_{n}(A(\delta))\right)^{1 / 2}
$$

and

$$
d(x, y) \leq 2\left(M_{n}(A(\delta))\right)^{1 / 2}|x-y|\left(\sum_{j=1}^{n}(\pi j)^{2}\right)^{1 / 2} \leq c\left(M_{n}(A(\delta))\right)^{1 / 2}|x-y| n^{3 / 2}
$$


for some constant $c>0$. In what follows, we write $c$ for any positive constant. Let $N(\varepsilon)=N([0,0.5], d ; \varepsilon)$ be the $\varepsilon$-covering number of $[0,0.5]$, that is, the minimal number of open balls of radius $\varepsilon>0$ in the pseudometric $d$ which is necessary to cover $[0,0.5]$. Then

$$
N(\varepsilon) \leq 1+\varepsilon^{-1} c n^{3 / 2}\left(M_{n}(A(\delta))\right)^{1 / 2} .
$$

By virtue of (3.3), Proposition 13.8 and Lemma 13.5 of Ledoux and Talagrand (1991), there exists an absolute constant $c>0$ such that

$$
\begin{aligned}
E_{G} \max _{x \in[0,0.5]}\left|\sum_{j=1}^{n}\left(A_{j}(\delta)\right)^{1 / 2} G_{j} \exp (-i 2 \pi x j)\right|^{2} \\
\quad \leq c E_{\varepsilon} \max _{x \in[0,0.5]}\left|\sum_{j=1}^{n}\left(A_{j}(\delta)\right)^{1 / 2} \varepsilon_{j} \exp (-i 2 \pi x j)\right|^{2} \\
\quad \leq c\left(D+\int_{0}^{D}(\ln N(\varepsilon))^{1 / 2} d \varepsilon\right)^{2} \\
\quad \leq c\left(2\left(S_{n}(A(\delta))\right)^{1 / 2}+\int_{0}^{D}(\ln N(\varepsilon))^{1 / 2} d \varepsilon\right)^{2}
\end{aligned}
$$

where $\left(\varepsilon_{j}\right)$ is a Rademacher sequence, independent of $\left(A_{j}\right)$, and $E_{G}$ and $E_{\varepsilon}$ denote expectations with respect to $G_{j}$ and $\left(\varepsilon_{j}\right)$, respectively [with $\left(A_{j}\right)$ fixed]. By (3.3) and (3.4) we have

$$
\begin{aligned}
\int_{0}^{D}(\ln N(\varepsilon))^{1 / 2} d \varepsilon \leq & \int_{0}^{2\left(S_{n}(A(\delta))\right)^{1 / 2}}\left(\ln \left(1+\varepsilon^{-1} c n^{3 / 2}\left(M_{n}(A(\delta))\right)^{1 / 2}\right)\right)^{1 / 2} d \varepsilon \\
= & c n^{3 / 2}\left(M_{n}(A(\delta))\right)^{1 / 2} \\
& \times \int_{0}^{2 c^{-1} n^{-3 / 2}\left(S_{n}(A(\delta)) / M_{n}(A(\delta))\right)^{1 / 2}}\left(\ln \left(1+\varepsilon^{-1}\right)\right)^{1 / 2} d \varepsilon .
\end{aligned}
$$

Using, for example, Karamata's theorem [cf. Bingham, Goldie and Teugels (1987)], one can check that for any $\theta>0$,

$$
\int_{0}^{\theta}\left(\ln \left(1+\varepsilon^{-1}\right)\right)^{1 / 2} d \varepsilon \leq c \theta\left(\ln \left(1+\theta^{-1}\right)\right)^{1 / 2} .
$$

Therefore,

$$
\int_{0}^{D}(\ln N(\varepsilon))^{1 / 2} d \varepsilon \leq c\left(S_{n}(A(\delta))\right)^{1 / 2}\left(\ln \left(1+c n^{3 / 2} \frac{\left(M_{n}(A(\delta))\right)^{1 / 2}}{\left(S_{n}(A(\delta))\right)^{1 / 2}}\right)\right)^{1 / 2}
$$

We conclude from (3.5) and (3.6) that

$$
E_{G} \max _{x \in[0,0.5]}\left|\sum_{j=1}^{n} G_{j}\left(A_{j}(\delta)\right)^{1 / 2} \exp (-i 2 \pi x j)\right|^{2} \leq c S_{n}(A(\delta)) \ln n .
$$


Now, by (3.7), (3.2) and an application of the Cauchy-Schwarz inequality,

$$
E\left(I_{n, Z}^{-}(\delta)\right)^{\alpha / 8} \leq c(\ln n)^{\alpha / 8} E\left(n^{-2 / \alpha} S_{n}(A(\delta))\right)^{\alpha / 8} .
$$

We have, by Jensen's inequality,

$$
E\left(n^{-2 / \alpha} S_{n}(A(\delta))\right)^{\alpha / 8} \leq\left(n^{1-2 / \alpha} E A_{1}(\delta)\right)^{\alpha / 8} .
$$

Direct calculation yields

$$
E A_{1}(\delta) \leq c \delta^{1-\alpha / 2} n^{2 / \alpha-1} .
$$

We conclude that

$$
(\ln n)^{-\alpha / 8} E\left(I_{n, Z}^{-}(\delta)\right)^{\alpha / 8} \leq c \delta^{(\alpha / 8)(1-\alpha / 2)} .
$$

Now take $\delta=\delta_{n}=(\ln n)^{-\beta}$ for some $\beta>0$ to be chosen later. Then it follows that the sequence

$$
(\ln n)^{\beta(1-\alpha / 2)-1} I_{n, Z}^{-}(\delta), \quad n \geq 1
$$

is tight.

Define now

$$
I_{n, Z}^{+}(\delta)=\max _{x \in[0,0.5]}\left|n^{-1 / \alpha} \sum_{j=1}^{n} G_{j}\left(A_{j}^{c}(\delta)\right)^{1 / 2} \exp (-i 2 \pi x j)\right|^{2},
$$

where

$$
A_{j}^{c}(\delta)=A_{j} I_{\left\{A_{j}>\delta n^{2 / \alpha}\right\}}
$$

We have

$$
I_{n, Z}^{+}(\delta) \leq\left|n^{-1 / \alpha} \sum_{j=1}^{n}\right| G_{j}\left|\left(A_{j}^{c}(\delta)\right)^{1 / 2}\right|^{2} .
$$

Clearly, there is a $C>1$ such that for all $M>1$,

$$
\left(M^{-1} A_{j} \mid A_{j}>M\right) \stackrel{\text { st }}{\leq} C U_{j}^{-2 / \alpha},
$$

where $\leq^{\text {st }}$ denotes stochastic domination, $U_{j}$ are iid uniform $U(0,1)$ random variables, independent of $\left(G_{j}\right)$ and of the binomial variable

$$
N_{n, \delta}=\#\left\{j: 1 \leq j \leq n ; A_{j}>\delta n^{2 / \alpha}\right\} .
$$

Choose as above $\delta=\delta_{n}=(\ln n)^{-\beta}$. Then

$$
n^{-1 / \alpha} \sum_{j=1}^{n}\left|G_{j}\right|\left(A_{j}^{c}(\delta)\right)^{1 / 2} \stackrel{\text { st }}{\leq} c(\ln n)^{-\beta / 2} \sum_{j=1}^{N_{n, \delta_{n}}}\left|G_{j}\right| U_{j}^{-1 / \alpha} .
$$

Furthermore, by simply taking the means we see that the sequence $\left(N_{n, \delta_{n}} /(\ln n)^{\alpha \beta / 2}\right)$ is tight. 
Now assume that $\alpha \in(1,2)$. By the law of large numbers, the sequence

$$
\left((\ln n)^{-\alpha \beta / 2} \sum_{j=1}^{N_{n, \delta_{n}}}\left|G_{j}\right| U_{j}^{-1 / \alpha}\right)
$$

is tight and, therefore, so is the sequence

$$
\left((\ln n)^{-\beta(\alpha-1)} I_{n, Z}^{+}(\delta)\right) .
$$

Combining this fact with (3.8) and choosing $\beta=2 / \alpha$, we conclude that the statement of the proposition holds in the case $\alpha \in(1,2)$.

Now we turn to the case $\alpha=1$. We know that

$$
\sum_{j=1}^{n}\left|G_{j}\right| U_{j}^{-1} \stackrel{d}{=} \Gamma_{n+1} \sum_{j=1}^{n}\left|G_{j}\right| \Gamma_{j}^{-1},
$$

where $\Gamma_{j}$ are the points of a unit rate Poisson process, independent of $\left(G_{j}\right)$. Recalling the rate of growth of the $\Gamma_{j}$ s, we easily see that the sequence

$$
(n \ln n)^{-1} \sum_{j=1}^{n}\left|G_{j}\right| U_{j}^{-1}, \quad n \geq 2
$$

is tight. This implies the tightness of the sequence

$$
(\ln n)^{-\beta / 2}(\ln \ln n)^{-1} \sum_{j=1}^{N_{n, \delta_{n}}}\left|G_{j}\right| U_{j}^{-1}, \quad n>e .
$$

We conclude that

$$
(\ln \ln n)^{-2} I_{n, Z}^{+}(\delta), \quad n>e
$$

is tight. Choosing now $\beta=2$, we conclude from (3.8) that $\left(M_{n, Z} /(\ln \ln n)^{2}\right.$, $n>e)$ is tight. This concludes the proof of the proposition in all cases.

As in the case $\alpha<1$ we also formulate a result for the linear process $\left(X_{t}\right)$ given by (1.10). We have the following analogue to Lemma 2.6.

LEMMA 3.2. Assume that the power transfer function $f_{X}$ is positive on $[0,0.5]$ and that the coefficients $\psi_{j}$ of the linear process $\left(X_{t}\right)$ satisfy the condition

$$
\sum_{j \neq 0, \pm 1}\left|\psi_{j}\right|(\ln |j|)^{(1 / \alpha)-0.5}<\infty
$$

if $1<\alpha<2$ and

$$
\sum_{j \neq 0, \pm 1}\left|\psi_{j}\right|(\ln |j|)^{1.5}(\ln |\ln | j||)^{-1}<\infty
$$


if $\alpha=1$. Then the following relation holds:

$$
\beta_{n}^{-2} \max _{x \in[0,0.5]}\left|I_{n, X}(x) / f_{X}(x)-I_{n, Z}(x)\right| \stackrel{P}{\rightarrow} 0,
$$

where

$$
\beta_{n}= \begin{cases}(\ln n)^{1-1 / \alpha}, & \text { if } \alpha \in(1,2), \\ \ln \ln n & \text { if } \alpha=1\end{cases}
$$

REMARK. Conditions (3.10) and (3.11) are stronger than (1.11) and (1.12). The latter restriction is needed in order to ensure the convergence of series (1.10). On the other hand, conditions (3.10) and (3.11) imply, in particular, that $f_{X}$ is continuous.

Proof. First recall the decompositions (2.19) and (2.20). By virtue of Proposition 3.1 it suffices to show that $\beta_{n}^{-1} \max _{x \in[0,0.5]}\left|Y_{n}(x)\right| \rightarrow^{P} 0$. As in the proof of Lemma 2.6 we assume for convenience that $\psi_{j}=0$ for $j<0$. Following the lines of the proof of Lemma 2.6 and using the same notation, we conclude from (3.10) or (3.11) and Proposition 3.1 that

$$
\beta_{n}^{-1} \max _{x \in[0,0.5]}\left|S_{11}(x)\right| \leq\left(\beta_{n}^{-2} M_{n, Z}\right)^{1 / 2} \sum_{j=n+1}^{\infty}\left|\psi_{j}\right| \stackrel{P}{\rightarrow} 0 .
$$

Furthermore, the same arguments as for (3.7) lead to the inequalities

$$
\begin{aligned}
E_{G} \max _{x \in[0,0.5]}\left|S_{12}(x)\right| & \leq n^{-1 / \alpha} \sum_{j=n+1}^{\infty}\left|\psi_{j}\right| E_{G} \max _{x \in[0,0.5]}\left|\sum_{t=1-j}^{n-j} G_{t} A_{t}^{1 / 2} \exp (-i 2 \pi x t)\right| \\
& \leq c \sum_{j=n+1}^{\infty}\left|\psi_{j}\right|\left(n^{-2 / \alpha} \ln n \sum_{t=1-j}^{n-j} A_{t}\right)^{1 / 2} \\
& \leq c(\ln n)^{1 / 2} \sum_{k=1}^{\infty} \psi_{n, k}\left(n^{-2 / \alpha} \sum_{j=-(k+1) n}^{-(k-1) n} A_{j}\right)^{1 / 2}
\end{aligned}
$$

where

$$
\psi_{n, k}=\sum_{j=k n+1}^{(k+1) n}\left|\psi_{j}\right|
$$

Suppose first that $1<\alpha<2$. Observe that the expression under the square root above has the distribution of $(2+1 / n)^{\alpha / 2} A_{1}$. Taking another expectation, we obtain by (3.11),

$$
\beta_{n}^{-1} E \max _{x \in[0,0.5]}\left|S_{12}(x)\right| \leq \beta_{n}^{-1} c(\ln n)^{1 / 2} \sum_{k=1}^{\infty} \psi_{n, k} \leq c \sum_{j=n+1}^{\infty}(\ln |j|)^{(1 / \alpha)-0.5}\left|\psi_{j}\right| \rightarrow 0 .
$$


Now suppose that $\alpha=1$, and rewrite the expression above as

$$
E_{G} \max _{x \in[0,0.5]}\left|S_{12}(x)\right| \leq c(\ln n)^{1 / 2} \sum_{k=1}^{\infty} \psi_{n, k}\left(B_{k}^{1 / 2}+\widetilde{B}_{k}^{1 / 2}\right),
$$

where $\left(B_{k}, k \geq 1\right)$ and $\left(\widetilde{B}_{k}, k \geq 1\right)$ are two (not independent) sequences of iid $S_{1 / 2}(1,1,0)$ random variables. To prove that $\beta_{n}^{-1} \max _{x \in[0,0.5]}\left|S_{11}(x)\right| \rightarrow^{P} 0$ it is, then, enough to prove that

$$
D_{n}=\beta_{n}^{-1}(\ln n)^{1 / 2} \sum_{k=1}^{\infty} \psi_{n, k} B_{k}^{1 / 2} \stackrel{P}{\rightarrow} 0 .
$$

Letting $\gamma_{n}=\left|\psi_{n}\right|(\ln n)^{1 / 2}(\ln \ln n)^{-1}$, we have

$$
D_{n} \leq \sum_{k=1}^{\infty}\left(\sum_{j=k n+1}^{(k+1) n} \gamma_{j}\right) B_{k}^{1 / 2} \stackrel{d}{=} \sum_{k=1}^{\infty}\left(\sum_{j=k n+1}^{(k+1) n} B_{j} \gamma_{j}^{2}\right)^{1 / 2} \leq \sum_{j=n+1}^{\infty} B_{j}^{1 / 2} \gamma_{j}
$$

and so (3.12) follows from (3.11) and the Three Series Theorem.

Using the same conditioning argument as above, one can show that

$$
\begin{aligned}
& E_{G} \max _{x \in[0,0.5]}\left|S_{2}(x)\right| \\
& \quad \leq c n^{-1 / \alpha} \sum_{j=1}^{n}\left|\psi_{j}\right|(\ln j)^{1 / 2}\left(\left(S_{j-1}(B)\right)^{1 / 2}+\left(S_{j-1}(\widetilde{B})\right)^{1 / 2}\right) \\
& \quad \leq c n^{-1 / \alpha} \sum_{j=1}^{n}\left|\psi_{j}\right|(\ln j)^{1 / 2}\left(\left(S_{n-1}(B)\right)^{1 / 2}+\left(S_{n-1}(\widetilde{B})\right)^{1 / 2}\right) \\
& \quad \stackrel{d}{=} c \sum_{j=1}^{n}\left|\psi_{j}\right|(\ln j)^{1 / 2}\left(B_{1}^{1 / 2}+\left(\widetilde{B}_{1}\right)^{1 / 2}\right)
\end{aligned}
$$

where

$$
S_{j}(B)=\sum_{k=0}^{j} B_{k} \quad \text { and } \quad S_{j}(\widetilde{B})=\sum_{k=0}^{j} \widetilde{B}_{k}, \quad j \geq 0
$$

and $\left(B_{k}, k \geq 1\right)$ and $\left(\widetilde{B}_{k}, l \geq 1\right)$ are two (this time independent) sequences of iid $S_{\alpha / 2}(1,1,0)$ random variables. By virtue of (3.10) and (3.11), it is easy to see that $\beta_{n}^{-1} \sum_{j=1}^{n}\left|\psi_{j}\right|(\ln j)^{1 / 2} \rightarrow 0$. Therefore, using (3.13), we finally conclude that

$$
\beta_{n}^{-1} E \max _{x \in[0,0.5]}\left|S_{2}(x)\right| \rightarrow 0
$$

in all cases. This concludes the proof of the lemma.

An argument very similar to that of Lemma 3.2 proves the following analogue of Corollary 2.7. 
COROLlaRY 3.3. Assume that $f_{X}$ is positive on $[0,0.5]$ and that the coefficients $\psi_{j}$ of the linear process $\left(X_{t}\right)$ satisfy the conditions $(3.10)$ if $1<\alpha<2$ and (3.11) if $\alpha=1$. Then the sequence $\beta_{n}^{-2} \max _{x \in[0,0.5]} I_{n, X}(x) / f_{X}(x)$ is tight.

Acknowledgments. This project commenced during a visit by Thomas Mikosch to the school of ORIE at Cornell University in the summer of 1997. He would like to express his gratitude to his colleagues for their excellent hospitality. The authors are grateful to the anonymous referee for careful reading of the paper. An error in an earlier version of the paper was corrected because of the referee's comments.

\section{REFERENCES}

Billingsley, P. (1968). Convergence of Probability Measures. Wiley, New York.

Bingham, N., Goldie, C. and Teugels, J. (1987). Regular Variation. Cambridge Univ. Press.

Brockwell, P. and Davis, R. (1991). Time Series: Theory and Methods, 2nd ed. Springer, New York.

Chen, Z.-G. and Hannan, E. (1980). The distribution of the periodogram ordinates. J. Time Ser. Anal. 1 73-82.

Davis, R. and Mikosch, T. (1999). The maximum of the periodogram of a non-Gaussian sequence. Ann. Probab. 27 522-536.

Feller, W. (1971). An Introduction to Probability Theory and Its Applications 2, 2nd ed. Wiley, New York.

Fisher, R. A. (1929). Tests of significance in harmonic analysis. Proc. Roy. Statist. Soc. Ser. A 125 54-59.

Freedman, D. and Lane, D. (1980). The empirical distribution of Fourier coefficients. Ann. Statist. 8 1244-1251.

Freedman, D. and LANE, D. (1981). The empirical distribution of the Fourier coefficients of a sequence of independent, identically distributed long-tailed random variables. Z. Wahrsch. Verw. Gebiete 58 21-39.

KLÜPpelberG, C. and Mikosch, T. (1993). Spectral estimates and stable processes. Stochastic Process. Appl. 47 323-344.

Ledoux, M. and TALAGRAND, M. (1991). Probability in Banach Spaces: Isoperimetry and Processes. Springer, New York.

Priestley, M. (1981). Spectral Analysis and Time Series I, II. Academic Press, New York.

RESNICK, S. (1986). Point processes. Regular variation and weak convergence. Adv. in Appl. Probab. 18 66-138.

Resnick, S. (1987). Extreme Values. Regular Variation and Point Processes. Springer, New York.

SAmorodnitsky, G. and TAqQU, M. (1994). Stable Non-Gaussian Random Processes. Chapman and Hall. New York.

TURKMAN, K. F. and WALKER, A. (1984). On the asymptotic distributions of maxima of trigonometric polynomials with random coefficients. Adv. in Appl. Probab. 16 819-842.

WEYL, H. (1916). Über die Gleichverteilung von Zahlen mod Eins. Math. Ann. 77 313-352.

\section{T. Mikosch}

DEPARTMENT OF MATHEMATiCs

P.O. BOX 800

UNIVERSITY OF GRONINGEN

NL-9700 AV GRONINGEN

THE NETHERLANDS
S. I. RESNICK

G. SAMORODNITSKY

School OF Operations RESEARCH AND INDUSTRIAL ENGINEERING

CORNELL UNIVERSITY

ITHACA, NEW YORK 14853

E-MAIL: gennady@orie.cornell.edu 